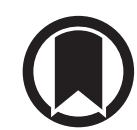

CrossMark

\section{The airway microbiota in children newly diagnosed with bronchiectasis largely retains its diversity}

\author{
To the Editor:
}

There is a great deal of interest in the airway microbiota, its diversity and the role of specific microbial taxa in the pathophysiology of lung disease [1]. Non-cystic fibrosis bronchiectasis is a significant public health problem in many countries, including New Zealand, where prevalence is high and morbidity and mortality are substantial [2-4]. A role for bacteria in the pathophysiology of bronchiectasis is widely accepted but poorly characterised due to inherent difficulties with lower airway sampling, especially in young children. Culture-based studies demonstrate associations with Haemophilus influenzae, Moraxella catarrhalis and Streptococcus pneumoniae in children [2,5], and Pseudomonas aeruginosa in adults and those with severe disease $[6,7]$. Culture-independent methods have revealed complex airway microbial communities with differences between children and adults with bronchiectasis, and between adults with cystic fibrosis and bronchiectasis [8]. A shared core microbiota was reported for children with bronchiectasis, protracted bacterial bronchitis (PBB) and cystic fibrosis, with most of these taxa also seen in healthy controls [8]. Subsequent research indicated that microbiota composition could be used to distinguish bronchiectasis, PBB and control children [9]. In children with PBB, bronchial brush samples were dominated by Haemophilus, Moraxella, Streptococcus and Neisseria [10], while bronchoalveolar lavage (BAL) samples showed that Bacteroides and Haemophilus were more common than in disease controls, with Lactococcus and Lactobacillus less common [11]. Differences in study findings could be due to methodological differences, disease heterogeneity and timing of airway samples. Adults with bronchiectasis demonstrate a dominance of either Haemophilus or Pseudomonas. Pseudomonal dominance is associated with end-stage lung disease and potentially adverse clinical outcomes [6, 7]. Recent longitudinal studies in children with cystic fibrosis demonstrate a reduction in microbial diversity with age, with establishment of more traditional disease-modifying taxa [12]. An emerging common theme is that reduced microbiota diversity, and certain bacterial taxa, may potentially be associated with adverse clinical outcomes.

We undertook a prospective study at Starship Children's Hospital, Auckland, New Zealand between 2015 and 2017, enrolling children being investigated for bronchiectasis as per our regional guidelines [13]. Flexible bronchoscopic BAL ( $1 \mathrm{~mL} \cdot \mathrm{kg}^{-1}$ lavage of $0.9 \%$ saline, maximum $\left.20 \mathrm{~mL}\right)$ was performed from the 2-3 most affected lobes in children with bronchiectasis as identified by chest computed tomography (CT) scan. Bronchiectasis was radiologically defined as a broncho-arterial ratio of more than 1.0 in combination with other well-described radiological features [2, 14]. We also recruited controls undergoing elective surgery, with no respiratory history. A single non-bronchoscopic lavage was performed in the controls. To assess potential contamination introduced from the bronchoscope, triplicate bronchoscopy negative controls were conducted. DNA was extracted using the Qiagen AllPrep DNA/RNA Mini Kit, and PCR-amplified bacterial 16S rRNA genes (V3-V4 region) sequenced using Illumina MiSeq [15]. Bacterial load was estimated using real-time PCR quantification of 16S rRNA gene copies. Negative (no-template) PCR controls and extraction kit controls using sterile water were performed.

Patient data were compared using a Chi-squared test for categorical variables and Kruskal-Wallis test for continuous variables. Sequence and statistical analyses utilised USEARCH, QIIME and R to calculate diversity metrics based on 97\% $16 \mathrm{~S}$ rRNA gene-defined bacterial operational taxonomic units (OTUs) [15].

@ERSpublications

Young children diagnosed with bronchiectasis have a lower airway microbiota that is highly diverse and very similar to healthy controls. Haemophilus, Moraxella, Neisseria and Streptococcus are the most abundant operational taxonomic units identified. http://bit.ly/2SkqRrq

Cite this article as: Pillarisetti $\mathrm{N}$, Broderick $\mathrm{D}$, Ainsworth $\mathrm{A}$, et al. The airway microbiota in children newly diagnosed with bronchiectasis largely retains its diversity. Eur Respir J 2019; 54: 1900704 [https:// doi.org/10.1183/13993003.00704-2019]. 

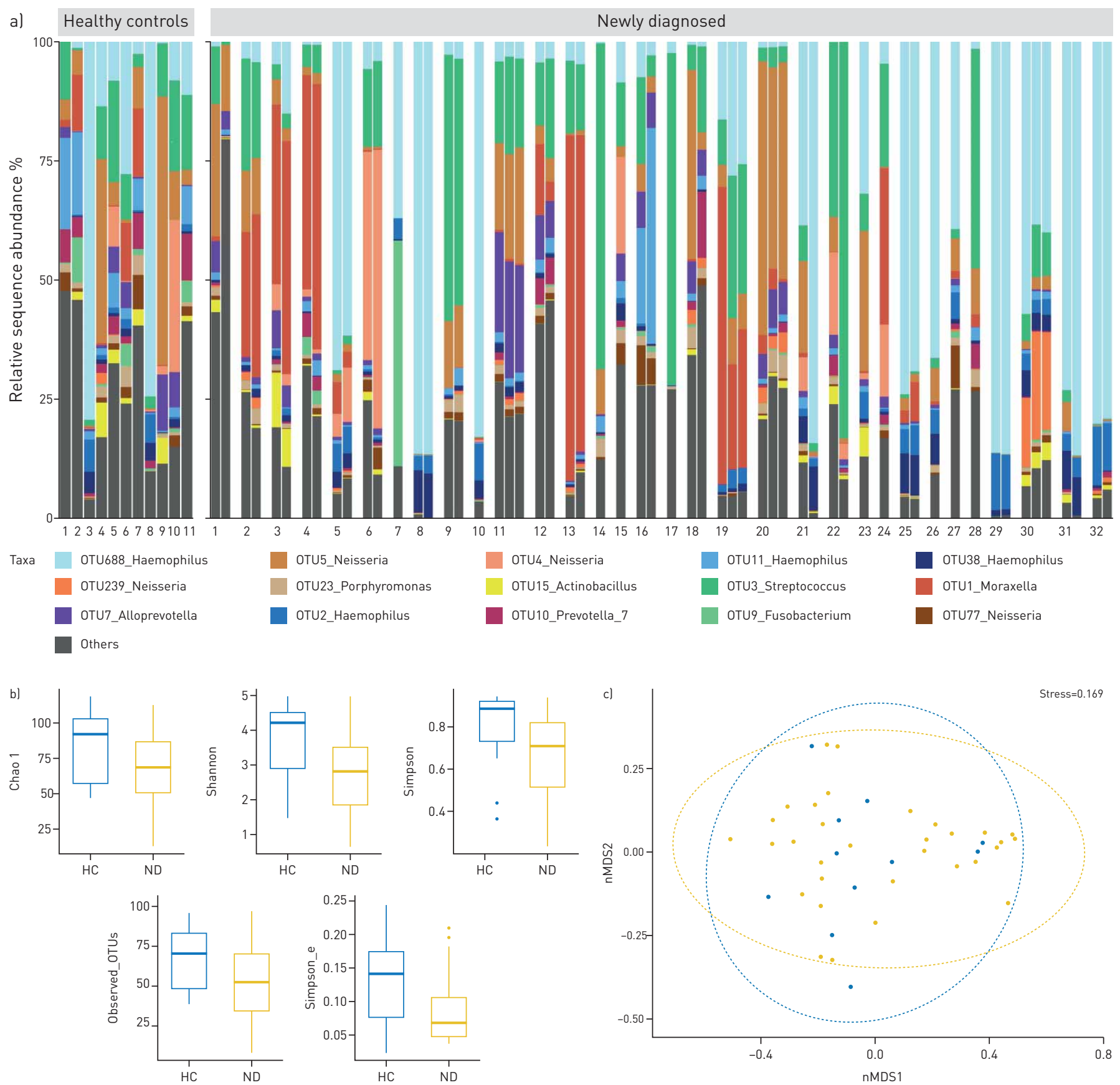

FIGURE 1 Bacterial community composition and diversity for newly diagnosed bronchiectasis (ND, $\mathrm{n}=32$ ) and healthy controls (HC, $\mathrm{n}=11$ ). a) Relative abundances of taxon-assigned operational taxonomic units (OTUs) at 97\% 16S rRNA gene sequence similarity in lavage samples taken from healthy controls and newly diagnosed bronchiectasis. The 15 most abundant OTUs are shown, with all remaining taxon-assigned OTUs grouped in "Others". b) Alpha diversity comparisons between cohorts. Median values are indicated by the solid line within each box, and the box extends to upper and lower quartile values; outliers are indicated by closed circles. c) Beta diversity visualised using non-metric multidimensional scaling (nMDS) of the Bray-Curtis dissimilarity metric. Ellipses represent the mean of the description coordinates at the centre, with dispersion of the ellipses calculated using the standard deviation of the weighted average of covariance matrix group scores.

Sequence data were deposited in the National Center for Biotechnology Information Sequence Read Archive (PRJNA 422718).

32 children (21 male and 11 female) with bronchiectasis (median age 2.3 years, range 0.9-16 years) and 11 (seven male and four female) healthy controls (median age 5.9 years, range 1.4-13.1 years) were recruited. There were significant differences $(\mathrm{p}<0.05)$ between groups, with the bronchiectasis cohort having an over-representation of Maori $(21,65.6 \%)$ and Pasifika $(6,18.8 \%)$ children, more tobacco smoke exposure and coming from areas of higher social deprivation, compared to controls. The aetiology of bronchiectasis 
after extensive work-up was post-infectious $(15,46.9 \%)$, idiopathic $(10,31.3 \%)$ and aspiration-related (5, $15.6 \%)$. Bilateral bronchiectasis was seen in $26(81.3 \%)$ children, with three or more affected lobes in 21 $(65.6 \%)$ children.

We obtained 2214195 high-quality 16S rRNA gene sequences in total, with 83 bacterial genera identified across 188 OTUs. Overall, Proteobacteria (mean \pm SD relative sequence abundance $62.6 \pm 28.8 \%$ ), Firmicutes $(21.1 \pm 22.5 \%)$ and Bacteroidetes $(9.4 \pm 8.6 \%)$ were the dominant bacterial phyla in children with bronchiectasis. The most abundant genera, which were frequently represented by multiple OTUs, were

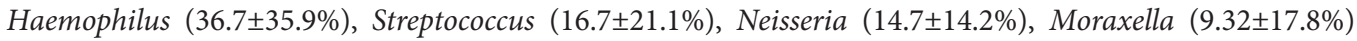
and Fusobacterium $(3.85 \pm 9.6 \%)$ (figure 1a). OTUs representing Haemophilus, Neisseria and Streptococcus were also prominent in healthy controls. Inter-individual differences in children with bronchiectasis were considerable, explaining $49 \%$ of observed microbiota variation (PERMANOVA, $\mathrm{R}^{2}=0.49, \mathrm{p}=0.001$ ), while intra-individual differences were minimal (figure 1a). Bacterial community richness and diversity did not differ significantly $(\mathrm{p}>0.05)$ between children with bronchiectasis and controls across multiple indices, namely Chao1, Shannon, Simpson's index, number of observed OTUs, and Simpson's evenness measure E (figure 1b). Non-metric multidimensional scaling plots reaffirmed the high degree of overlap in microbiotas of controls and children with bronchiectasis (figure 1c), while Dunn's test with Bonferroni and false discovery rate corrections revealed no significant differences in OTU relative sequence abundance between the cohorts. Real-time PCR indicated no significant difference in lower airway bacterial load between groups (Wilcoxon rank sum test $\mathrm{p}>0.05$ ).

Consistent with the small number of studies in children, Haemophilus, Streptococcus, Moraxella and Neisseria were the most abundant taxa in lower airways of our children with bronchiectasis. Measures of alpha and beta diversity were also similar between children with bronchiectasis and healthy controls. This is in contrast with adults with bronchiectasis who additionally harbour Pseudomonas and Staphylococcus [7]. A study of bacterial communities in resected lung tissue demonstrated that, in advanced disease, the microbiota of bronchiectasis was similar to that of cystic fibrosis, with Proteobacteria being the most dominant phylum and Pseudomonas being the most prevalent genus [6]. Thus, current evidence suggests that airway microbiota in some airway diseases and especially bronchiectasis is dynamic and evolves over time. This, and the association of microbiota and disease outcomes need further evaluation.

One limitation of our study is the relatively small number of children involved, although our numbers are comparable to other recent studies and reflect challenges in obtaining paediatric lower airway samples. The strengths of this study include prospective recruitment of children, targeted lower airway sampling in children with CT-diagnosed bronchiectasis and healthy controls, and successful characterisation of the lower airway microbiota in a condition for which few data are available. While one recent study showed that a combination of nasopharyngeal and oropharyngeal sampling provided a representation of the lower airway microbiota, upper airway samples nonetheless still differed significantly from BAL samples [9]. Thus, in our opinion, lavage remains the gold standard for accurately studying lower airway disease. Importantly, bronchoscopy controls, extraction and no-template PCR controls yielded a negligible number of $16 \mathrm{~S}$ rRNA gene sequences, giving us confidence in the integrity of our obtained data.

Our data suggest that, at the point of diagnosis of bronchiectasis, lower airway bacterial diversity is largely preserved, similar to that of healthy controls, and changes associated with progressive disease are not yet present. If this diversity can continue to be maintained, we speculate that this could limit disease progression. Future studies, preferably incorporating a longitudinal aspect, should focus on disease progression in relation to microbiota changes, as well as the function of the microorganisms in question.

Naveen Pillarisetti ${ }^{1,2}$, David Broderick ${ }^{3}$, Alana Ainsworth ${ }^{1,2}$, Anna Mulholland ${ }^{1,2}$, Brett Wagner Mackenzie ${ }^{4}$, Danielle Middleton $^{3}$, Catherine A. Byrnes ${ }^{1,2}$ and Michael W. Taylor ${ }^{3}$

${ }^{1}$ Dept of Paediatric Respiratory Medicine, Starship Children's Hospital, Auckland, New Zealand. ${ }^{2}$ Dept of Paediatrics and Child Health, University of Auckland, Auckland, New Zealand. ${ }^{3}$ School of Biological Sciences and Maurice Wilkins Centre for Molecular Biodiscovery, University of Auckland, Auckland, New Zealand. ${ }^{4}$ Dept of Surgery, University of Auckland, Auckland, New Zealand.

Correspondence: Naveen Pillarisetti, Paediatric Respiratory Medicine, Starship Children’s Hospital, Park Road, Grafton, Auckland 1023, New Zealand. E-mail: naveenp@adhb.govt.nz

Received: Feb 072019 | Accepted after revision: April 162019

Acknowledgments: We are very grateful to the children and parents who participated in the study. We also acknowledge the support of the anaesthesia team, respiratory team, operating theatre and support staff of Starship Children's Hospital and the Lab Plus services. We would like to thank our funding bodies: the Athlae Lyon Starship Research Trust, A+ Trust and Starship Foundation. 
Author contributions: N. Pillarisetti, C.A. Byrnes and M.W. Taylor conceived and designed the study. N. Pillarisetti, A. Ainsworth and A. Mulholland were responsible for the recruitment and sampling of individuals. D. Broderick and D. Middleton analysed the samples. D. Broderick and B. Wagner Mackenzie conducted the bioinformatics analyses. All authors contributed to manuscript writing and editing.

Support statement: Supported by Athlae Lyon Starship Research Trust, A+ Trust, Starship Foundation. Funding information for this article has been deposited with the Crossref Funder Registry.

Conflict of interest: None declared.

\section{References}

1 Faner R, Sibila $\mathrm{O}$, Agusti A, et al. The microbiome in respiratory medicine: current challenges and future perspectives. Eur Respir J 2017; 49: 1602086.

2 Twiss J, Metcalfe R, Edwards E, et al. New Zealand national incidence of bronchiectasis “too high" for a developed country. Arch Dis Child 2005; 90: 737-740.

3 Asthma and Respiratory Foundation of New Zealand. Te Hā Ora (The Breath of Life): National Respiratory Strategy. Wellington, The Asthma Foundation, 2015.

4 Chang AB, Bush A, Grimwood K. Bronchiectasis in children: diagnosis and treatment. Lancet 2018; 392: 866-879.

5 Flume PA, Chalmers JD, Olivier KN. Advances in bronchiectasis: endotyping, genetics, microbiome, and disease heterogeneity. Lancet 2018; 392: 880-890.

6 Maughan H, Cunningham KS, Wang PW, et al. Pulmonary bacterial communities in surgically resected noncystic fibrosis bronchiectasis lungs are similar to those in cystic fibrosis. Pulm Med 2012; 2012: 746358.

7 Rogers GB, van der Gast CJ, Cuthbertson L, et al. Clinical measures of disease in adult non-CF bronchiectasis correlate with airway microbiota composition. Thorax 2013; 68: 731-737.

8 van der Gast CJ, Cuthbertson L, Rogers G, et al. Three clinically distinct chronic pediatric airway infections share a common core microbiota. Ann Am Thorac Soc 2014; 11: 1039-1048.

9 Marsh RL, Kaestli M, Chang AB, et al. The microbiota in bronchoalveolar lavage from young children with chronic lung disease includes taxa present in both the oropharynx and nasopharynx. Microbiome 2016; 4: 37.

10 Cuthbertson L, Craven V, Bingle L, et al. The impact of persistent bacterial bronchitis on the pulmonary microbiome of children. PLoS One 2017; 12: e0190075.

11 Bao Y, Li Y, Qiu C, et al. Bronchoalveolar lavage fluid microbiota dysbiosis in infants with protracted bacterial bronchitis. J Thorac Dis 2018; 10: 168-174.

12 Frayman KB, Armstrong DS, Carzino R, et al. The lower airway microbiota in early cystic fibrosis lung disease: a longitudinal analysis. Thorax 2017; 72: 1104-1112.

13 Chang AB, Bell SC, Torzillo PJ, et al. Grimwood K, extended voting group. Chronic suppurative lung disease and bronchiectasis in children and adults in Australia and New Zealand Thoracic Society of Australia and New Zealand guidelines. Med J Aust 2015; 202: 21-23.

14 Hansell DM. Bronchiectasis. Radiol Clin North Am 1998; 36: 107-128.

15 Hoggard M, Biswas K, Zoing M, et al. Evidence of microbiota dysbiosis in chronic rhinosinusitis. Int Forum Allergy Rhinol 2017; 7: 230-239. 